Research Article

\title{
A re-annotation of the Anopheles darlingi mobilome
}

\author{
Jose Francisco Diesel ${ }^{1}$, Mauro F. Ortiz ${ }^{2}$, Osvaldo Marinotti ${ }^{3}$, Ana Tereza R. Vasconcelos ${ }^{4}$ and Elgion L. S. \\ Loreto $^{1}$ iD \\ ${ }^{1}$ Departamento de Bioquímica e Biologia Molecular, Universidade Federal de Santa Maria, Santa Maria, \\ RS, Brazil. \\ ${ }^{2}$ Programa de Pós-Graduação de Genética e Biologia Molecular, Universidade Federal do Rio Grande do \\ Sul, Porto Alegre, RS, Brazil. \\ ${ }^{3}$ Department of Molecular Biology and Biochemistry, University of California at Irvine, Irvine, CA, USA. \\ ${ }^{4}$ Laboratório de Bioinformática do Laboratório Nacional de Computação Científica, Petrópolis, RJ, Brazil.
}

\begin{abstract}
The mobilome, portion of the genome composed of transposable elements (TEs), of Anopheles darlingi was described together with the genome of this species. Here, this mobilome was revised using similarity and de novo search approaches. A total of $5.6 \%$ of the A. darlingi genome is derived of TEs. Class I gypsy and copia were the most abundant superfamilies, corresponding to $22.36 \%$ of the mobilome. Non-LTR elements of the $R 1$ and Jockey superfamilies account for $11 \%$ of the TEs. Among Class II TEs, the mariner superfamily is the most abundant (16.01\%). Approximately $87 \%$ of the A. darlingi mobilome consist of short, truncated and/or degenerated copies of TEs. Only three retrotransposons, two belonging to gypsy and one to copia superfamilies, are putatively active elements. Only one Class II element, belonging to the mariner superfamily, is putatively active, having 12 copies in the genome. The TE landscape of $A$. darlingi is formed primarily by degenerated elements and, therefore, somewhat stable. Future applications of TE-based vectors for genetic transformation of $A$. darlingi should take into consideration mariner and piggyBac transposons, because full length and putatively active copies of these elements are present in its genome.
\end{abstract}

Keywords: Transposable elements, transposons, mosquitoes, evolvability.

Received: September 19, 2017; Accepted: April 19, 2018.

\section{Introduction}

The mobilome is the complete set of mobile genetic elements in a genome. In eukaryotes, it is constituted mainly by transposable elements (TEs) (Siefert, 2009), comprising about $45 \%$ of the human genome, $20 \%$ of $D$. melanogaster, and more than $50 \%$ of the maize genome (SanMiguel et al., 1996; Lander et al., 2001; Kaminker et al., 2002). Transposable elements are drivers of evolution, as a source of genetic variability, generally by promoting chromosome rearrangements, mutations in the coding or regulatory regions of genes, domestication and epigenetic alterations (reviewed in Hua-Van et al., 2011).

TEs are classified as autonomous elements when they are able to produce the enzymes necessary for their own mobilization, or as non-autonomous when they require enzymes produced by related autonomous elements for that activity. TEs have also been classified into two classes,

Send correspondence to Elgion L. S. Loreto. Department of Biochemistry and Molecular Biology, CCNE, Federal University of Santa Maria, Av. Roraima 1000, Camobi, 97105-900 Santa Maria, RS, Brazil. E-mail: elgion@ base.ufsm.br. namely RNA-mediated (Class-I) and DNA-mediated (Class-II) elements, according to their transposition mode. TEs often occur as remains or relics of old elements, which are not mobilizable any more. The combination of active, mobilizable, and remnants of TEs constitutes the TE landscape of a genome, which is characteristic of a species. For example, in humans, the LINE-1/L1-element is the only element that is presently active, while in Drosophila melanogaster $30 \%$ of the TEs are full length and potentially active (Kaminker et al., 2002). Closely related species can display distinct TE contents. For instance, TE contents vary from 2.7 to $23 \%$ among the genomes of 12 Drosophila species (Clark et al., 2007), and among Anopheles species it varies from 1.98 to $17.78 \%$ (Neafsey et al., 2015). Yet, a full, integral insight into the mobilome is not provided just by the proportion of a genome occupied by TEs and their classification. Also important is the identification of full length, putatively active elements. This aspect is particularly important for organisms that are potential candidates for genetic manipulation using transposon-based transgenic technologies. In these cases, the characterization of full 
length and active transposable elements is fundamental to estimate genomic stability and biosafety of the proposed products (Terenius et al., 2008). In genetically transformed organisms, the presence of active endogenous TEs similar to the one(s) used in the transformation vector(s) might interfere with the efficiency of transgene integration and transgene stability due to cross mobilization (Arensburger et al., 2011).

The classification and annotation of TEs is always a challenging task due to their remarkable diversity within and among genomes. TE copies recently inserted into a genome show low sequence variability, though with time passing, copies accumulate mutations, deletions, and/or insertions, becoming decayed TE remnants (Hua-Van et al., 2011; Hoen et al., 2015). Two main approaches are currently used for TEs identification and annotation. Homology-based methods search for sequences similar to known TEs compiled in databases. The de novo approach is based on the search for repetitiveness and structural signatures normally found in TEs (Hoen et al., 2015). New tools for mobilome scrutiny, exploration, and annotation warrant the re-analysis of previously described genomes (Kaminker et al., 2002). Fernández-Medina et al. (2011) who reanalyzed the mobilome of $A$. gambiae, found new TEs, described complete and potentially active elements, and characterized additional deleted, mutated, and probably inactive copies.

Anopheles darlingi is the principal Neotropical malaria vector, responsible for more than a million malaria cases per year (Oliveira-Ferreira et al., 2010). The genome of this mosquito was sequenced, annotated, and its mobilome described (Marinotti et al., 2013). In that study, TEs were annotated applying a homology-based method, using a "home-made" TE database. De novo search was used only to find MITEs and SINEs. In the present study, the Repbase database was used for a homology-based search, and the Repeatscout program was used for de novo searches. These improved approaches allowed us to advance our knowledge of the A. darlingi mobilome, and to revise the number and annotation of the identified TEs in its genome.

\section{Material and Methods}

A. darlingi transposable elements were identified following the pipeline shown in Figure 1. Blastn and tblastx (Altschul et al., 1997) were used to find similarities of $A$. darlingi genome sequences (GenBank accession number ADMH02000000) (Marinotti et al., 2013) with the TEs references of the Repbase database (version 18.01) (Jurka et $a l$. , 2005), considering e-values $<10 \mathrm{e}-10$ as a cutoff to define a Blast "hit". Redundancies representing hits at overlapping genomic positions, for different TEs, were considered as one hit for further analyses. Each target was expanded $5 \mathrm{~kb}$ on each side and searched for TIRs, LTR, TSD and conserved ORFs with the UGENE platform (Okonechnikov et al., 2012). The Censor software (Kohany et

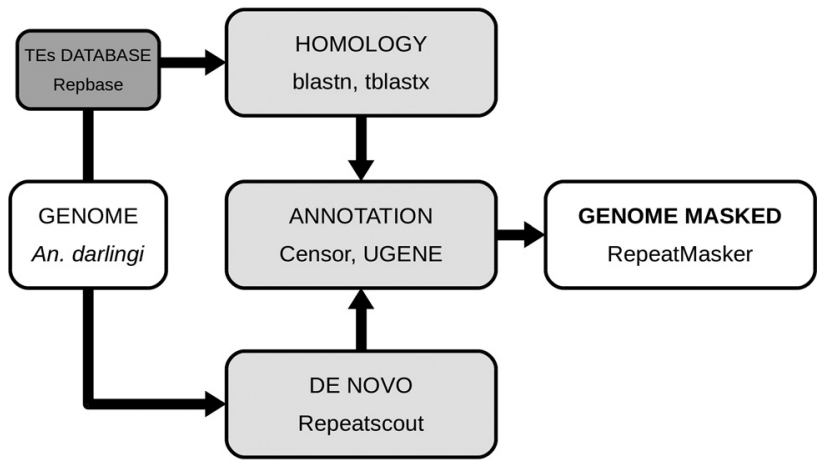

Figure 1 - Flowchart depicting the pipeline implemented in this study for analysis and annotation of the An. darlingi mobilome. The assembled genome (ADMH02000000) was screened for TEs by similarity-based approach, using the Repbase database by Blastn and tblastx. In parallel, the genome was screened de novo by using Repeatscout. Redundancies were removed and TEs were classified using Censor with the Repbase database. Also, a manual annotation was performed, using UGENE, with emphasis on TIRs, LTRs, TSDs and ORFs. The content of TEs in the genome was calculated using RepeatMasker.

al., 2006) implemented with the Repbase database was used for the classification and annotation of TEs. For de novo searches, Repeatscout version 1.0.0 software (Price et $a l ., 2005)$ was used. The obtained sequences were analyzed using UGENE to look for characteristics described previously (TIRs, LTRs, etc.) and to classify these transposable elements. After obtaining a full library by similarities and de novo searches, the genome was masked to determine the number of transposable elements using RepeatMasker (with -no_is -nolow options) (Smit et al., 2016). TEs were classified using Repbase (DNA, ERV, LTR, Non-LTR), and their number of copies and $\%$ of genome were calculated.

Many short retrieved sequences show similarities with known TEs sequences. These short sequences are mentioned in the text as "hits" and classified as belonging to the superfamily corresponding to the best blast hit (lowest e-value). Sequences longer than $200 \mathrm{bp}$ were manually curated with the UGENE platform for the annotation of ORFs, TIR, LTRs, and TSDs. All ORFs were analyzed by Blastp, and those showing similarities with TEs-encoded proteins were manually annotated. The sequences with typical TE characteristics were designated as identifiable TEs (ITEs) and classified as: (1) complete elements - containing TIR (or LTRs) and complete ORFs; (2) degenerated - most often contain TIRs (or LTRs), however, the ORFs present mutations and deletions; (3) truncated - these elements show large deletions; (4) MITEs - short elements, having TIRs. When possible, sequences were assigned into families using Repbase; otherwise they were described as Unknown.

The repeat landscape of TEs found in the A. darlingi genome was constructed with the full TE dataset obtained using the RepeatMasker tool. 


\section{Results}

The TEs identified in this study correspond to $5.6 \%$ of the $A$. darlingi genome (Table 1). Class I elements correspond to $2.64 \%$ and Class II to $2.44 \%$ of the genome. For Class I, the superfamilies gypsy and copia were the most abundant. Together, these superfamilies account for $22.36 \%$ of all TEs. Non-LTR elements of $R 1$ and Jockey superfamilies compound, together, $11 \%$ of all TEs. The mariner superfamily is the most abundant among the Class II elements (16.01\%). Endogenous retroviruses (ERVs) correspond to $8.23 \%$ of the TEs.

A remarkable aspect of the $A$. darlingi mobilome is that it is composed mainly of very short sequences displaying significant similarities with TEs present in the used database. These hits are likely derived from extensively mutated and/or deleted TEs, lacking clearly identifiable TE structural features such as TIRs, LTRs, or TSD, etc.. The sequences containing identifiable TE structural features, ITEs, correspond to only $0.87 \%$ of the $A$. darlingi genome (Table 2). This value was obtained by multiplying the size of each element by copy number, which corresponds to 1.5 $\mathrm{Mb}$. The entire A. darlingi genome was estimated to be
173.9 Mb (Marinotti et al., 2013). Thirty-six ITE elements were found, 26 belonging to the mariner family, one to the piggyBac family, one to kolobok family, two elements are from the gypsy family, one from the copia family, and four DNA/Unknown elements (Table 2). Short descriptions and sequences of each element are presented in List S1 of the Supplementary Material.

mariner elements are predominantly degenerated, and 17 elements were classified with this status. The copy number of these degenerated mariner elements range from 2 to 200 copies, and their sizes vary from 1755 to $889 \mathrm{bp}$. Six mariner elements were classified as MITEs, ranging from 489 to $796 \mathrm{bp}$, and are represented by 1 to 49 copies. Two mariner elements were classified as truncated. One putatively active mariner element was identified. It is represented by 12 copies in the genome, has a length of 1664 $\mathrm{bp}$, and long TIRs with $230 \mathrm{bp}$.

Four degenerated elements were classified as DNA/Unknown because they have characteristics of class II elements, but their similarities with known TEs are not high enough for their classification into known families. The copy number of these elements is generally high, rang-

Table 1 - Sequences showing significant hits with TEs identified in Anopheles darlingi genome.

\begin{tabular}{|c|c|c|c|c|c|c|}
\hline Class & Order & Superfamily & Hit number & Occupied size (bp) & TE $\%$ & Genome \% \\
\hline \multirow[t]{11}{*}{ I $(2.64 \%)$} & \multirow[t]{5}{*}{ LTR $(1.40 \%)$} & Gypsy & 18007 & 1304425 & 11.27 & 0.75 \\
\hline & & Copia & 17890 & 1283868 & 11.09 & 0.74 \\
\hline & & BEL & 1262 & 114186 & 0.98 & 0.06 \\
\hline & & DIRS & 740 & 47004 & 0.41 & 0.03 \\
\hline & & Others & 2589 & 159289 & 1.37 & 0.09 \\
\hline & \multirow[t]{6}{*}{ Non-LTR (1.25\%) } & $\mathrm{R} 1$ & 7744 & 654295 & 5.65 & 0.37 \\
\hline & & Jockey & 7685 & 614572 & 5.31 & 0.35 \\
\hline & & Tx1 & 4074 & 302983 & 2.62 & 0.17 \\
\hline & & L1 & 3944 & 256751 & 2.22 & 0.15 \\
\hline & & SINE2/tRNA & 2645 & 199096 & 1.72 & 0.11 \\
\hline & & Others & 7100 & 589270 & 5.09 & 0.34 \\
\hline \multirow[t]{6}{*}{ II $(2.44 \%)$} & & Mariner/Tc1 & 11871 & 1852562 & 16.01 & 1.06 \\
\hline & & hAT & 11167 & 723088 & 6.23 & 0.41 \\
\hline & & EnSpm/CACTA & 6961 & 474890 & 4.10 & 0.27 \\
\hline & & Polinton & 4207 & 268339 & 2.32 & 0.15 \\
\hline & & Helitron & 2552 & 186142 & 1.61 & 0.11 \\
\hline & & Others & 21620 & 1580047 & 13.65 & 0.91 \\
\hline \multirow[t]{5}{*}{ ERVs $(0.53 \%)$} & & ERV1 & 5835 & 441984 & 3.82 & 0.25 \\
\hline & & ERV2 & 5562 & 383990 & 3.32 & 0.22 \\
\hline & & ERV3 & 670 & 48140 & 0.41 & 0.03 \\
\hline & & ERV4 & 68 & 4117 & 0.03 & 0.01 \\
\hline & & Others & 1279 & 83874 & 0.72 & 0.04 \\
\hline TOTAL & & & 145472 & 11572912 & 100 & 6.65 \\
\hline TOTAL* & & & & & & 5.67 \\
\hline
\end{tabular}

*without redundancy 
Table 2 - Identifiable TEs found in the An. darlingi genome.

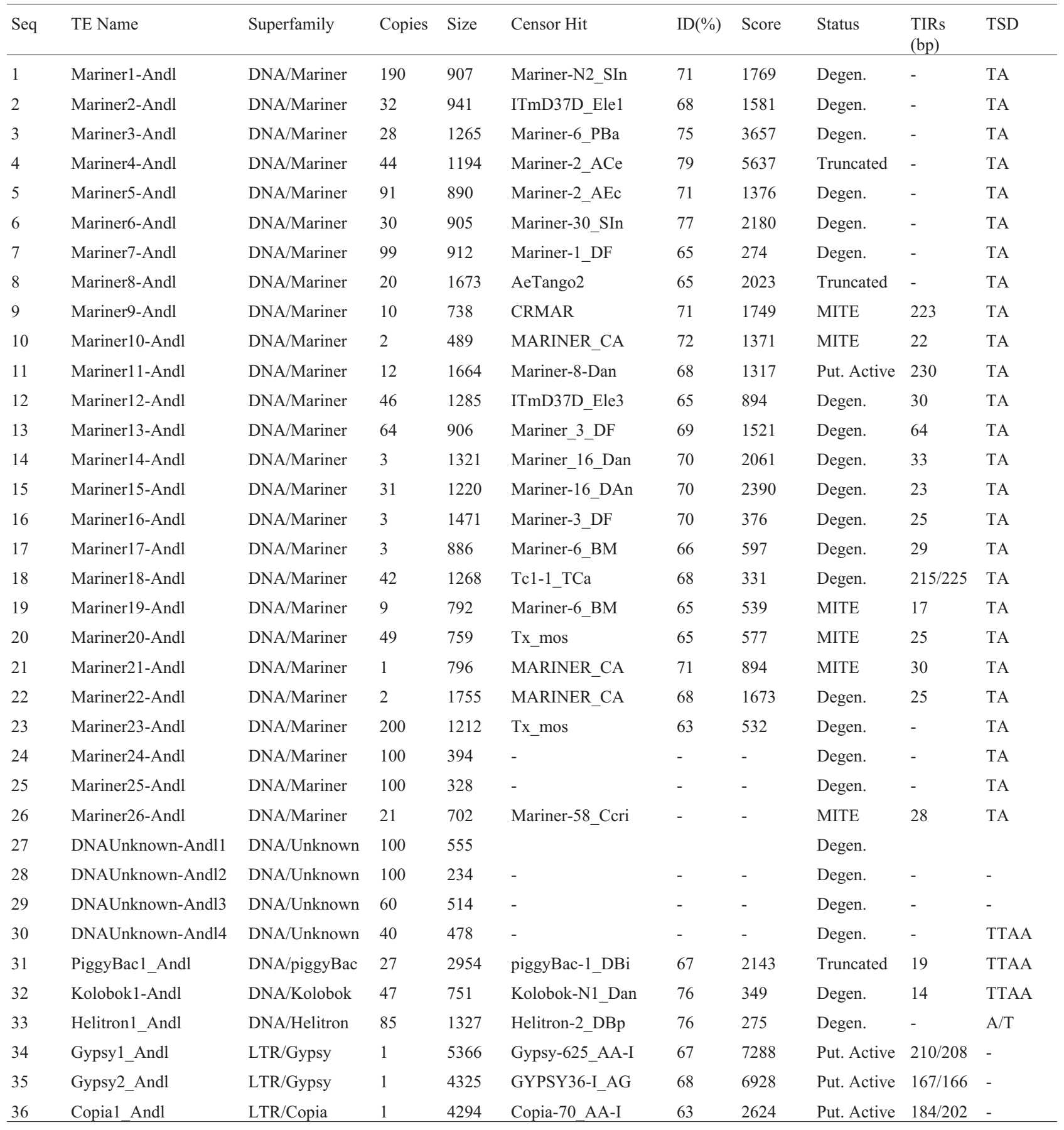

ing from 40 to more than 100 copies in the genome. Their sizes range from 234 to $555 \mathrm{bp}$, suggesting they are truncated elements.

A truncated piggyBac element of 2954 bp with a 19 bp TIR was found, with a total of 27 copies. Degenerated kolobok and helitron elements were also found, with 47 and 85 copies, respectively.

Only three Class I putatively active ITEs were found. Two elements are from the gypsy family, having lengths of
5366 and $4325 \mathrm{bp}$, with LTRs of 210 and $167 \mathrm{bp}$, respectively. The third element, belonging to the copia family, is 4294 bp in length and has LTRs with 184/202 bp. Only one copy of each of these retrotransposons was found in the $A$. darlingi genome.

Analysis of nucleotide divergence among the different copies of the found elements allowed to depict a general landscape of the mobilome. The level of Kimura substitution observed among the analyzed sequences is generally 
high (Figure 2). Few copies of the elements are well conserved, indicating that only few elements are active or have been recently mobilized or duplicated. So the mobilome of A. darlingi is constituted mainly of remains of degraded and ancient elements. In comparison, the proportion of copies showing high similarities is greater in $A$. gambiae than in $A$. darlingi (Figure 2), suggesting the presence of active or more recently mobilized elements in $A$. gambiae.

\section{Discussion}

The results of this study show that a higher proportion of the A. darlingi genome (5.61\%) is occupied by TEs than the previously reported $2.29 \%$ (Marinotti et al., 2013). For other organisms, re-analyses of mobilomes using updated tools and databases also resulted in distinct results. For example, the genomes of Drosophila species and A. gambiae have been re-analyzed resulting in improved descriptions of their mobilomes (Kaminker et al., 2002; Fernández-
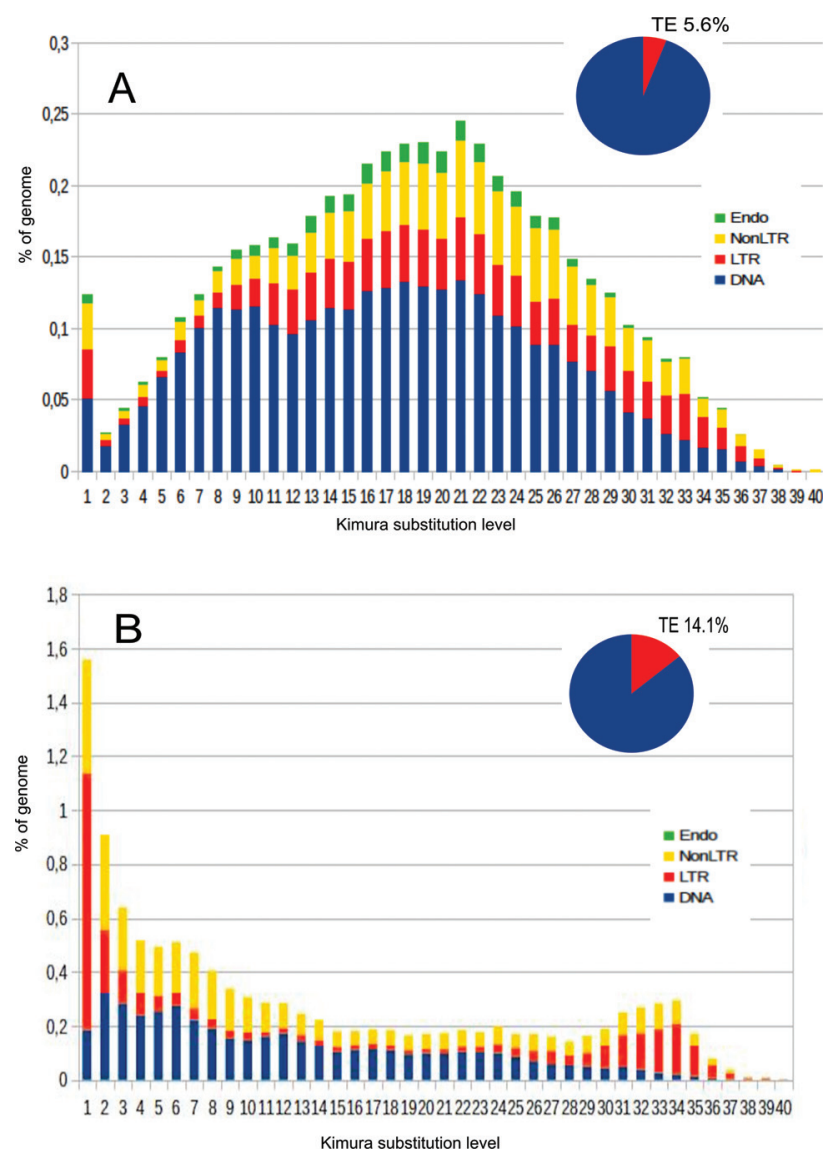

Figure 2 - Comparison of TE landscapes of $A$. darlingi (A) and $A$. gambiae (B). The pie charts show the proportion of the genome that is occupied by TEs (The value represented by the blue slice corresponds to portion that is not TEs). In the bar charts, the ordinate illustrates the percentage of the genome occupied by each TE, and the abscissa illustrates the genetic divergence from the consensus (Kimura substitution level- K2P x 100) observed among copies of each TE. Each TE superfamily is color coded. The landscapes were generated using www.repeatmasker.org/genomicDatasets/RMGenomicDatasets.html.
Medina et al., 2011, Neafsey et al., 2015). Neafsey et al. (2015) compared the genomes of 16 Anopheles species and found that $A$. albimanus was the mosquito with the genome occupied by the lowest proportion of TEs (1.98\%), followed by $A$. cristyi (2.81\%). The Anopheline species with the highest content of TEs in its genome is A. gambiae $(17.78 \%)$. The authors also reported a direct correlation between genome size and TE content, with species showing smaller genomes tending to have lower TE contents. Similar correlations between genome size and TE content have also been found for other taxa (Sessegolo et al., 2016). The genome size of $A$. darlingi is among the smallest among the sequenced Anopheles species, and similar to those found in A. albimanus and $A$. cristyi $[\approx 180 \mathrm{Mb}]$ (Table $\mathrm{S} 1$ ). While the TE content found in those species is around $2 \%$, our re-analyses increased the $A$. darlingi TE content from $2.29 \%$ to $5.61 \%$, a value comparable to those of Anopheline mosquitoes with larger genome sizes $(\approx 220 \mathrm{Mb})$. However, the direct correlation between TE content and genome size has outliers. For example, although $A$. quadriannulatus and $A$. gambiae have genomes with similar sizes, the TE content of the former is less than half of that of the latter (Neafsey et al., 2015, see also Table S1). Although there is variation, in Anopheline mosquitoes, Class I TEs are generally more abundant then those of Class II, as observed also in the present analysis for An. darlingi (Table S2).

The increased $A$. darling $i$ TE content reported in this study, compared to the previous description (Marinotti et al., 2013; Neafsey et al., 2015) is the result of an approach that utilized improved tools and databases. The database used for homology searches in the present study is larger than the one applied in the previous analysis. Also, it is due to the inclusion, in the present report, of short fragments derived from degenerated TEs. The overall proportional representations of the different TE superfamilies in $A$. darlingi were maintained between the present and previous analyses (Marinotti et al., 2013), with gypsy as the most abundant LTR element and mariner elements as the most abundant ones among the DNA transposons. However, some differences were seen for other superfamilies. For example, copia was the second most abundant superfamily observed in this study, but only $0.9 \%$ of copia elements were registered in the Marinotti et al. (2013) study.

Active or mobilizable elements are associated with evolvability of species and their capacity to environmental adaptation (Fablet and Vieira, 2011; Casacuberta and González, 2013). The TE landscape of $A$. darlingi is predominantly formed by degenerated elements, contrasting with species such as $A$. gambiae and D. melanogaster, which harbor a larger number of potentially active elements. In Drosophila melanogaster, $80 \%$ of spontaneous mutations are promoted by TE mobilizations, making it an important source of genetic variability (García Guerreiro, 2012). In contrast, only four putatively active elements were found in 
the assembled $A$. darlingi genome. It is also remarkable that the copy number found for putative active retrotransposon is very low; only one copy of each element has been identified. Only the putative active mariner element (Mariner11-Andl) has a higher copy number (12 copies). Judging from this landscape structure, the genome of $A$. darlingi is likely to be stable. Few TEs are capable of, or prone to respond to environmental stressors and likely to promote an increase in mutability.

As a recommendation for future uses of transposable elements as vectors for genetic transformation of $A$. darlingi, attention should be given to the use of mariner elements, as it was the only putatively active DNA TE found in the sequenced genome. piggyBac elements also deserve certain attention, because a truncated element is present in the genome. The use of other Class II transposable elements is suggested as being safer, as functional elements are not present to promote cross mobilization.

In conclusion, the mobilome of $A$. darlingi is primarily occupied by degenerated elements, showing a minute number of active elements with small copy number, characteristic of a genome that is rather stable.

\section{Acknowledgments}

This study was supported by research grants and fellowships from $\mathrm{CNPq}$ (Conselho Nacional de Desenvolvimento Científico e Tecnológico).

\section{Conflict of Intererest}

The authors have declared that no competing interests exist.

\section{Author contributions}

ELSL, MFO, ATRV and OM conceived and designed the study; JFD and MFO conducted the bioinformatics analysis; ELSL, JDD, MFO, ATRV and OM wrote the manuscript. All authors read and approved the final version.

\section{References}

Altschul SF, Madden TL, Schäffer AA, Zhang J, Zhang Z, Miller W and Lipman DJ (1997) Gapped BLAST and PSI-BLAST: A new generation of protein database search programs. Nucleic Acids Res 25:3389-3402.

Arensburger P, Hice RH, Wright JA, Craig NL and Atkinson PW (2011) The mosquito Aedes aegypti has a large genome size and high transposable element load but contains a low proportion of transposon-specific piRNAs. BMC Genomics 12:606.

Casacuberta E and González J (2013) The impact of transposable elements in environmental adaptation. Mol Ecol 22:15031517.

Clark AG, Eisen MB, Smith DR, Bergman CM, Oliver B, Markow TA, Kaufman T, Kellis M, Gelbart W, Iye VN et al.
(2007) Evolution of genes and genomes on the Drosophila phylogeny. Nature 450:203-218.

Fablet M and Vieira C (2011) Evolvability, epigenetics and transposable elements. BioMol Concepts 2:333-341.

Fernández-Medina RD, Struchiner CJ and Ribeiro JM (2011) Novel transposable elements from Anopheles gambiae. BMC Genomics 12:260.

García-Guerreiro MP (2012) What makes transposable elements move in the Drosophila genome? Heredity 108:461-468.

Hoen DR, Hickey G, Bourque G, Casacuberta J, Cordaux R, Feschotte C, Fiston-Lavier AS, Hua-Van A, Hubley R and Kapusta A et al. (2015) A call for benchmarking transposable element annotation methods. Mobile DNA 6:13.

Hua-Van A, Le Rouzic A, Boutin TS, Fillee J and Capy P (2011) The struggle for life of the genome's selfish architects. Biol Direct 6:19.

Jurka J, Kapitonov VV, Pavlicek A, Klonowski P, Kohany O and Wali-Chiewicz J (2005) Repbase update, a database of eukaryotic repetitive elements. Cytogenet Genome Res 110:462-467.

Kaminker JS, Bergman CM, Kronmiller B, Carlson J, Svirskas R, Patel S, Frise E, Wheeler DA, Lewis SE, Rubin GM et al. (2002) The transposable elements of the Drosophila melanogaster euchromatin: A genomics perspective. Genome Biol 3:1-20.

Kohany O, Gentles AJ, Hankus L and Jurka J (2006) Annotation, submission and screening of repetitive elements in Repbase: RepbaseSubmitter and Censor. BMC Bioinformatics 25:474.

Lander ES, Linton LM, Birren B, Nusbaum C, Zody MC, Baldwin J, Devon K, Dewar K, Doyle M, FitzHugh W et al. (2001) Initial sequencing and analysis of the human genome. Nature. 409:860-921.

Marinotti O, Cerqueira GC, de Almeida LG, Ferro MI, Loreto EL, Zaha A, Teixeira SM, Wespiser AR, Almeida E, Silva A et al. (2013) The genome of Anopheles darlingi, the main Neotropical malaria vector. Nucleic Acids Research 41:73877400.

Neafsey DE, Waterhouse RM, Abai MR, Aganezov SS, Alekseyev MA, Allen JE, Amon J, Arcá B, Arensburger P, Artemov G et al. (2015) Mosquito genomics. Highly evolvable malaria vectors: The genomes of 16 Anopheles mosquitoes. Science 347:1258522.

Okonechnikov K, Golosova O and Fursov M (2012) The UGENE team. Unipro UGENE: A unified bioinformatics toolkit. Bioinformatics 28:1166-1167.

Oliveira-Ferreira J, Lacerda MV, Brasil P, Ladislau JL, Tauil PL and Daniel-Ribeiro CT (2010) Malaria in Brazil: An overview. Malar J 9:115.

Price AL, Jones NC and Pevzner PA (2005) De novo identification of repeat families in large genomes. Bioinformatics Suppl 1:i351-8.

SanMiguel P, Tikhonov A, Jin YK, Motchoulskaia N, Zakharov D, Melake-Berhan A, Springer PS, EdwardsKJ, Lee M, Avramova Z et al. (1996) Nested retrotransposons in the intergenic regions of the maize genome. Science 274:765768.

Sessegolo C, Burlet N and Haudry A (2016) Strong phylogenetic inertia on genome size and transposable element content among 26 species of flies. Biol Lett 12:20160407. 
Siefert JL (2009) Defining the mobilome. Methods Mol Biol 532:13-27.

Smit AFA, Hubley R and Green P (2016) RepeatMasker Open4.0, http://www.repeatmasker.org.

Terenius O, Marinotti O, Sieglaff D and James AA (2008) Molecular genetic manipulation of vector mosquitoes. Cell Host Microbe 4:418- 423.

\section{Supplementary material}

The following online material is available for this article:
Table S1 - Comparison of genome size and TE contents in Anopheles genus.

Table S2 - Percentage of genome occupied by main TEs superfamilies in Anopheles genus.

List S1 - Description of 36 Identifiable TEs found in the Anopheles darlingi genome.

Associate Editor: Carlos F.M. Menck

License information: This is an open-access article distributed under the terms of the Creative Commons Attribution License (type CC-BY), which permits unrestricted use, distribution and reproduction in any medium, provided the original article is properly cited. 